\title{
INTEGRACION DE UN SIG CON MODELOS DE CALCULO Y OPTIMIZACION DE RUTAS DEVEHICULOS CVRP Y SOFTWARE DE GESTION DE FLOTAS
}

\author{
ALEJANDRO RODRIGUEZ VILLALOBOS \\ DEPARTAMENTO DE ORGANIZACION DE EMPRESAS \\ ESCUELA POLITECNICA SUPERIOR DE ALCOY \\ UNIVERSIDAD POLITECNICA DEVALENCIA
}

\begin{abstract}
Resumen: En esta comunicación se presentan los resultados alcanzados en un proyecto de I+D+I (Proyecto Rutas) cuyo principal objetivo es el desarrollo de una herramienta informática profesional que resuelva problemas reales de flotas de vehículos capacitados CVRP, el cálculo de rutas, y su gestión.
\end{abstract}

Palabras clave: Flotas, rutas, optimización, redes, SIG.

\section{Los problemas de rutas de vehículos (VRP)}

Los problemas de rutas de vehículos o de distribución física de mercancías desde almacenes a clientes aparecen en la literatura científica como Vehicle Routing Problems, más comúnmente como VRP.También se puede encontrar, aunque en menor medida, referencias como Vehicle Scheduling Problems. En términos generales, un problema de rutas de vehículos consiste en determinar las rutas de un conjunto (o flota) de vehículos que deben iniciar un recorrido (y finalizarlo) en los almacenes (o depósitos) para atender la demanda de servicio de un conjunto disperso de clientes sobre una red. Como se verá a continuación, las diferentes características de los clientes, la demanda, los almacenes y los vehículos, así como de las restricciones operativas sobre las rutas, horarios, etc. dan lugar a gran número de variantes del problema. En la literatura, algunos autores han in- tentado clasificar y simplificar la gran variedad de posibles problemas, como por ejemplo los criterios propuestos por (Bodin y Golden, 1981) y (Desrochers et al., 1990); que intentan reflejar y ordenar las principales características en aspectos como: el almacén o depósito, la flota, la demanda, el servicio y el objetivo a alcanzar. Esta clasificación de los problemas, ha facilitado tanto el desarrollo de modelos matemáticos y estrategias de resolución, como la toma de decisiones por parte de las empresas

\section{I.I. La red de transporte}

La red de carreteras o servicio utilizada para el transporte de bienes, se describe generalmente como un grafo donde los arcos representan los segmentos o secciones de las vías, y los vértices corresponden a las uniones o nodos de la red. En algunos casos los clientes o los depósitos pueden estar situados en dichos nodos, mientras que en otros casos pueden estar localizados en un arco del grafo. Los arcos (y por consiguiente el grafo) puede ser dirigidos o no dirigidos, dependiendo de si pueden ser circulados en un único sentido o en ambos (por ejemplo, calles de una única dirección o de ambos sentidos de circulación). Cada arco tendrá asociado un coste que puede representar su longitud en distancia, el tiempo de viaje, o el coste monetario del mismo. Alguno de estos parámetros pueden a su vez depender del tipo 
de vehículo o del momento en el que se recorra este arco (por ejemplo, las condiciones del tráfico en un momento dado).

\section{I.2. Los clientes y su servicio}

Cada cliente tendrá cierta necesidad de servicio o demanda que deberá ser atendida por algún vehículo. Es común que la demanda sea la necesidad de un conjunto de productos que ocupan volumen y peso en los vehículos, y como la capacidad de transporte del vehículo es limitada, es usual que un mismo vehículo no pueda satisfacer la demanda de todos los clientes. El servicio a los clientes no siempre implica distribuir producto desde el almacén hacia ellos, también puede entenderse que los clientes son proveedores, y por tanto se trataría de recoger mercancía para aprovisionar un almacén. En el caso de tratarse de una necesidad de servicio, el cliente simplemente debe ser visitado por el vehículo. Un mismo vehículo podría (en teoría) visitar a todos los clientes. El servicio requerido por el cliente podría ser también el de ser transportado hacia otra ubicación (servicio de transporte). En muchas ocasiones se trata de visitar al cliente exactamente una vez, sin embargo, en otros casos puede aceptarse que su demanda pueda ser atendida de manera fragmentada o por vehículos diferentes. Los clientes podrían tener restricciones de horario, en forma de intervalos o ventanas de tiempo dentro de las cuales se debe atender su servicio. También podría tenerse en cuenta no sólo el tiempo de recorrido por la red, sino el tiempo de servicio al cliente (carga y descarga).

También podrían existir restricciones de asociación entre vehículos y clientes, de manera que determinados clientes sólo puedan ser atendidos por determinados vehículos (por ejemplo, vehículos grandes y pesados que no pueden circular por calles estrechas o el centro urbano).

\section{I.3. Los almacenes o depósitos}

Tanto los productos a transportar (si los hubiera) como los vehículos, suelen estar localizados en los depósitos (almacenes, centros de tránsito, muelles o cocheras). Es habitual que las rutas den comienzo y/o finalicen en dichos depósitos. Aunque en algunos casos es diferente (por ejemplo, el viaje debe finalizar donde pernocta o finaliza la jornada el conductor). Pueden existir varios almacenes o depósitos con localización y otras características diferenciadoras (capacidad máxima de servicio o producción, horario, flota en origen, etc.). La flota asociada al depósito puede ser conocida o parte del objetivo a determinar. Debido al tiempo y espacio necesario para preparar y gestionar los vehículos, podría darse el caso de limitar el número de vehículos que operan a la vez en un mismo depósito (congestión de muelles).

\section{I.4. La flota de vehículos}

Los vehículos se definen por un conjunto de atributos, como su capacidad de carga en peso, en volumen, sus costes asociados, etc. En un vehículo se pueden transportar diferentes tipos de productos o uno sólo, asimismo su contenedor podría estar compartimentado o no. En la utilización de un vehículo se incurre en unos costes fijos por uso, y variables en función del tiempo, distancia u otros parámetros. Cuando los vehículos comparten unas mismas características se dice que la flota es homogénea, y si son diferentes flota heterogénea. El número de vehículos disponibles de una flota puede ser un dato conocido o una variable de decisión. Es común que el objetivo sea intentar utilizar la menor cantidad de vehículos y en segundo lugar minimizar la distancia o tiempo empleado de su ruta. La legislación o los convenios laborales pueden imponer restricciones sobre el tiempo máximo que un vehículo debe estar en circulación (descanso o relevo de conductores), su velocidad y carga máxima, e incluso el paso por determinadas zonas de la red. Es interesante en ocasiones intentar equilibrar las cargas de trabajo de los conductores, el tiempo o carga de los vehículos.

\subsection{Las rutas}

Los problemas de rutas de vehículos tratan por tanto de determinar la ruta o rutas para cada uno de los vehículos de la flota cumpliendo con todo el conjunto de restricciones e intentando alcanzar los objetivos propuestos. La función objetivo puede ser por ejemplo: minimizar los costes fijos, minimizar los costes totales, minimizar el número de vehículos requeridos, minimizar el tiempo total de transporte y/o la distancia total recorrida, minimizar las esperas, maximizar el beneficio de la operación, maximizar la función de utilidad del cliente, o su beneficio y satisfacción. En general en la literatura se asume que un vehículo sólo recorrerá una ruta en el período de planificación, pero también se pueden encontrar modelos en los que un mismo vehículo podría participar de más de una ruta. La siguiente Figura I (elaboración propia) representa un ejemplo típico de solución a un problema de rutas. En la figura se pue- 
Figura I

Ejemplo de la solución a un problema básico VRP (elaboración propia)

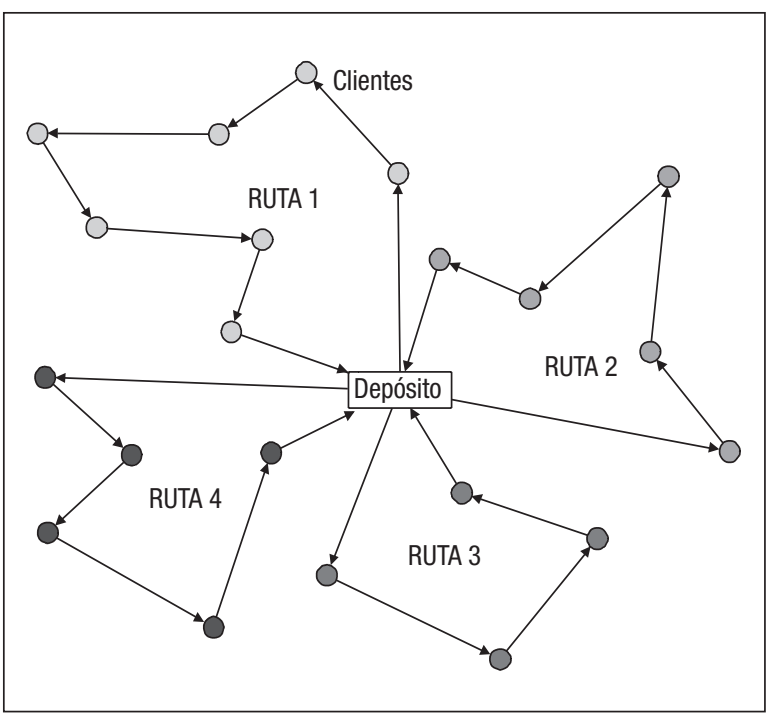

de observar 4 rutas diferentes con origen y destino final en el depósito central. Los arcos de la ruta solución deben ser necesariamente arcos de la red de transporte.

Como se ha visto anteriormente, los problemas de rutas son en realidad un amplio y complejo abanico de casos. Por otro lado, y tal y como subraya (Yepes, 2002), un caso real se define como resultado de la combinación de varias de estas características. El propio conjunto y variedad de características origina por explosión combinatoria y enorme número de posibles problemas (cada uno con su casuística concreta).

\section{La integración logística}

El desarrollo que se presenta en esta comunicación tiene como objetivos: facilitar la resolución de problemas reales de flotas de vehículos capacitados CVRP, el cálculo de rutas, y su gestión. En este desarrollo informático se integra inteligentemente tres elementos (Figura 2): el sistema de información geográfica SIG, la información del sistema logístico (VRP-XML), los modelos matemáticos y técnicas de optimización combinatoria que conjuntamente permiten resolver los problemas de rutas para flotas de vehículos, Toth et al. (200I pág. I-26).

El éxito en la gestión logística depende de la capacidad de integración (información y sistemas, proveedores y clientes, recursos y decisiones, etc.). Por ello, en este proyecto se ha prestado especial interés a la integración necesaria para la optimización del transporte, la toma de decisiones y la gestión de flotas.

\subsection{Desarrollo y estructura}

La herramienta informática desarrollada se ha estructurado de un modo abierto pero integrado. Todo el código fuente ha sido programado en Microsoft Visual Studio .NET, esto permite integrar fácilmente módulos y nuevas funciones programadas en diferentes lenguaje de programación. NET, a la vez que garantiza su funcionamiento en nuevas plataformas como Windows Vista, Windows 64-bit, Windows Mobile. La estructura del programa es modular, flexible y escalable (Figura 3). La programación orientada a objetos, y su estructura basada en clases y li-

Figura 2

Pantallas del software desarrollado para el cálculo de rutas y la gestión de flotas

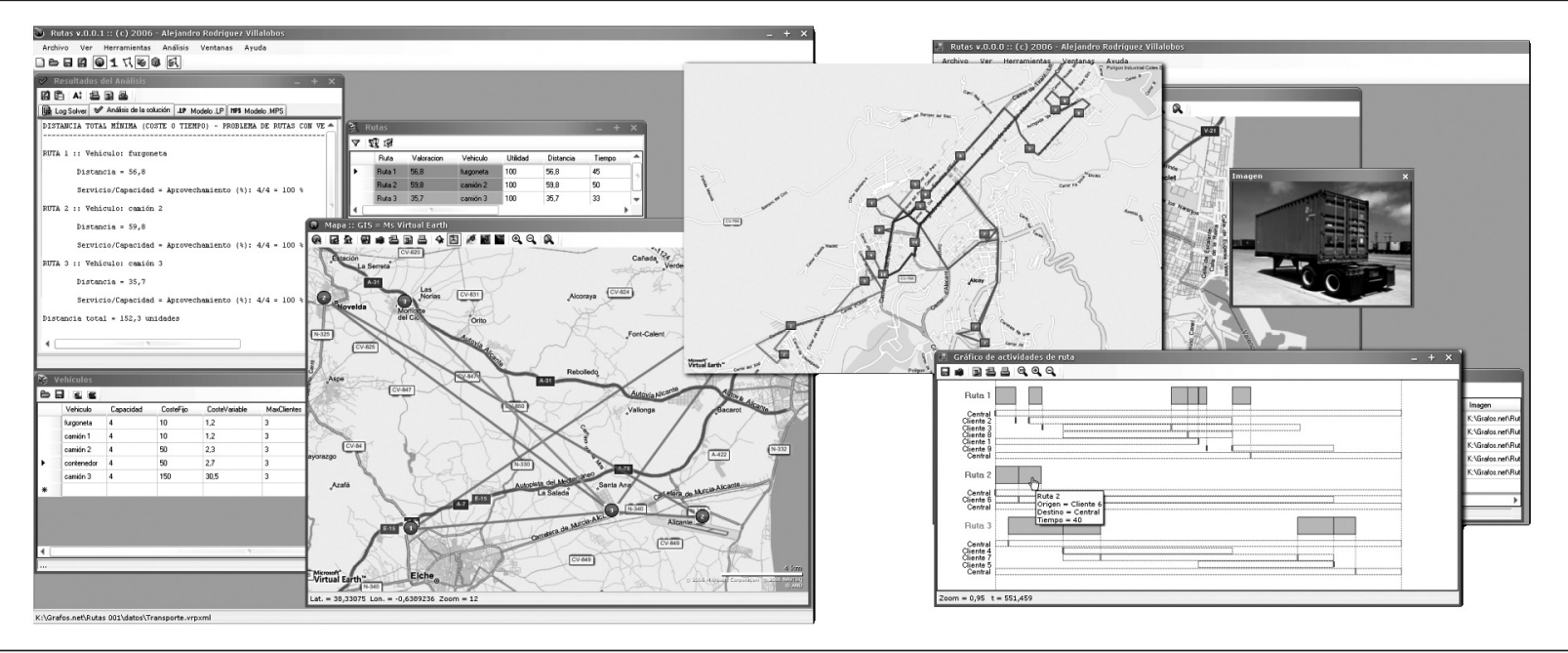


Figura 3

Estructura y principales componentes del proyecto rutas

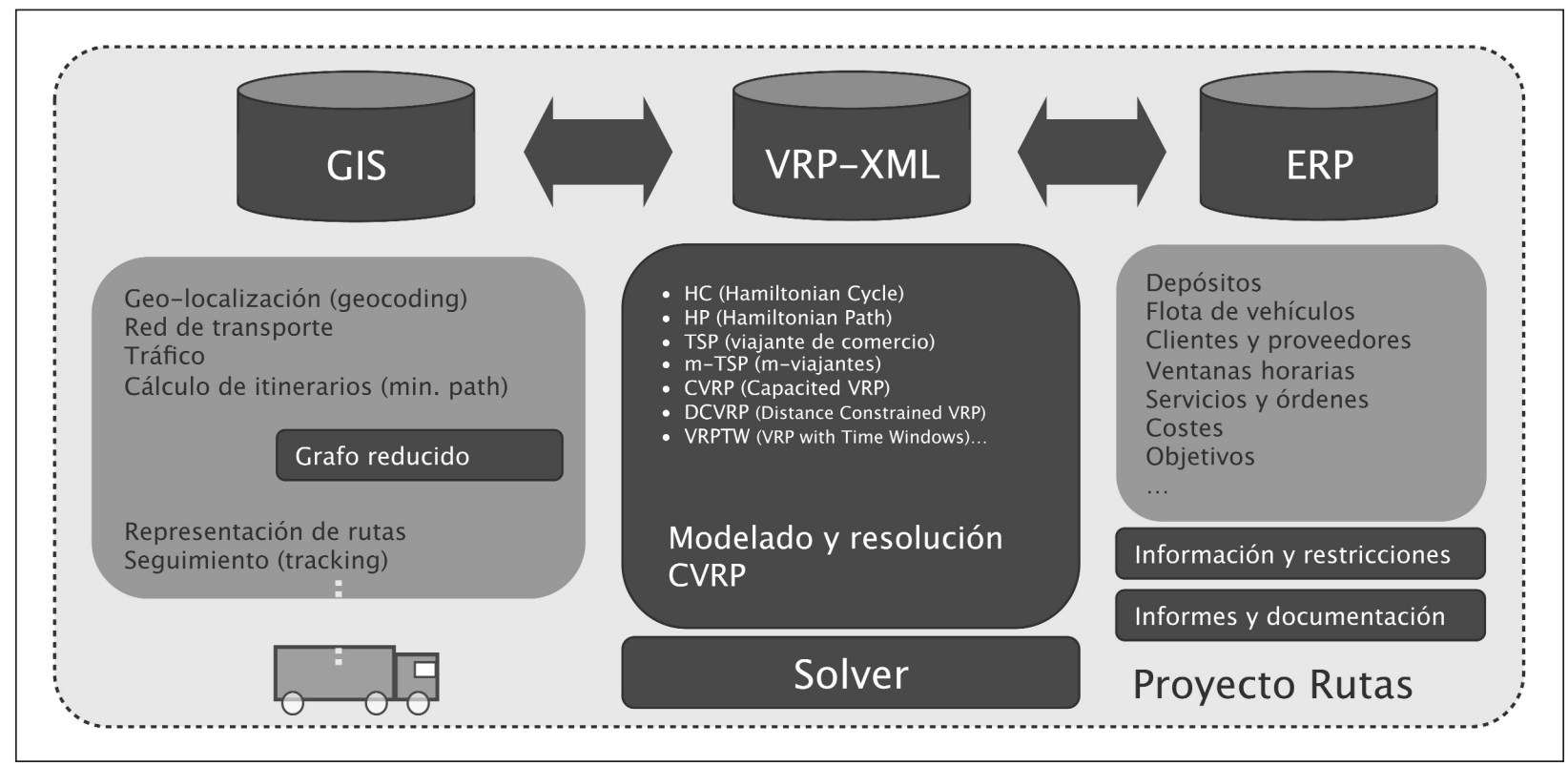

brerías (.dIl), permite fácilmente incorporar nuevas funciones y análisis, o mejorar los existentes.

Como se puede observar en la siguiente figura la herramienta combina e integra tres elementos: el sistema de información geográfica SIG, la información del sistema logístico (VRP-XML) y toda la información del sistema empresarial necesaria para crear y resolver los modelos matemáticos mediante técnicas de optimización combinatoria.

\subsubsection{El sistema de información geográfico (SIG)}

EI SIG es una colección organizada de datos geográficos que permite analizar y desplegar en todas sus formas la información geográficamente referenciada. En este caso el SIG se utiliza para las siguientes funciones:

- Geo-localización (geocoding) de los depósitos, clientes y proveedores.

- Análisis de la red de transporte, red de carreteras, sentido de circulación de las vías, tráfico, etc.

- Cálculo de itinerarios, caminos mínimos (en tiempo y/o distancia).

- Representación y seguimiento (tracking) de las rutas para cada vehículo.

El modo en el que se realiza la conectividad con el sistema de información geográfica (SIG), su vínculo con el cálculo, el análisis y la gestión del proceso logístico es una de las aportaciones clave en este trabajo. A diferencia de otro software existente en el mercado, éste no trabaja con información de toda la red SIG, sino que a partir de la información VRP-XML y tras un proceso de consultas se construye un grafo reducido que sólo contiene la información de la red necesaria en el proceso de modelado y resolución del problema CVRP. Esto mejora notablemente la eficiencia de la gestión de la información y reduce los tiempos computacionales necesarios.

\subsubsection{VRP-XML y el sistema de información empresarial}

Para el modelado, resolución y análisis de este tipo de problemas, es necesario gestionar una enorme cantidad de información: datos sobre las características de la flota de vehículos, los planes de ruta, los cargamentos, depósitos y recogidas, información geográfica, las restricciones y la función objetivo, etc. Tal y como se explica en Rodríguez (2006), se trata de una estructura de etiquetas VRP-XML que define los elementos de un documento que facilita el intercambio de datos en el contexto de los VRP (Vehicle Routing Problems). Todavía no existe un estándar consolidado para el intercambio de datos en este ámbito de trabajo, pero la estructura propuesta ha demostrado su validez en la aplicación empresarial de este proyecto. El módulo VRP-XML se enlaza fácilmente con el sistema de información empresarial (ERP), compartiendo datos de: clientes, servicios y 
órdenes de trabajo, información sobre costes de operaciones, disponibilidad e información sobre los recursos logísticos (flota de vehículos), ventanas horarias y otro tipo de restricciones, etc. Además este tipo de problemas son dinámicos y cambian en el tiempo, sus datos deben de estar soportados por una estructura flexible, capaz no sólo de atender tal cantidad de información según los actuales requerimientos de la empresa, sino también los futuros del sistema logístico (ampliación del número de clientes, de la flota de vehículos, nuevas restricciones, etc.).

\subsubsection{Modelado y resolución CVRP}

Como es conocido, los problemas CVRP son complejos de modelar y de resolver, ya que pertenecen al tipo de problemas NP-completo. El gestor y decisor del sistema logístico demanda una herramienta (Figura 4) que le haga transparente el proceso de modelado y optimización (o cálculo de soluciones factibles), pero que en cambio le permita explorar con detalle la bondad de la soluciones ayudándole en su toma de decisiones y le facilite la gestión (órdenes de trabajo, control, etc.).

Para poder resolver la gran variedad de tipos de problema VRP, el programa reúne y combina diferentes heurísticas y modelos de optimización. El usuario podrá elegir el tipo de análisis a realizar (Tabla I) y el software automáticamente validará la integridad de los datos y el problema planteado. Hay que subrayar que una de las aportaciones más importantes de este proyecto ha sido el desarrollo de las rutinas de modelado, y del código fuente necesario para la resolución de los problemas de programación lineal entera mixta. Esto es, el programa es capaz de realizar de manera transparente al usuario y en pocos instantes, el modelo de programación lineal necesario para la resolución y el análisis del problema, que el usuario haya definido en el grafo y en su estructura de meta-datos.

El problema CVRP básico trata de determinar los recorridos de $k$ vehículos de capacidad $C_{k}$ que partiendo de un origen común deben pasar por un conjunto de lugares de interés (clientes) para recoger o distribuir mercancías según una demanda $d_{i}$, y volver de nuevo al origen de manera que la distancia total recorrida (el coste o el tiempo empleado) por el conjunto de vehículos sea mínima. A continuación se muestra el modelo de tres subíndices [I].

Para un conjunto i, j de nodos del grafo, se expresa la función objetivo que intentará minimizar el coste total de todos los arcos recorridos en la solución. La

Figura 4

Integración de la información en el software Rutas.

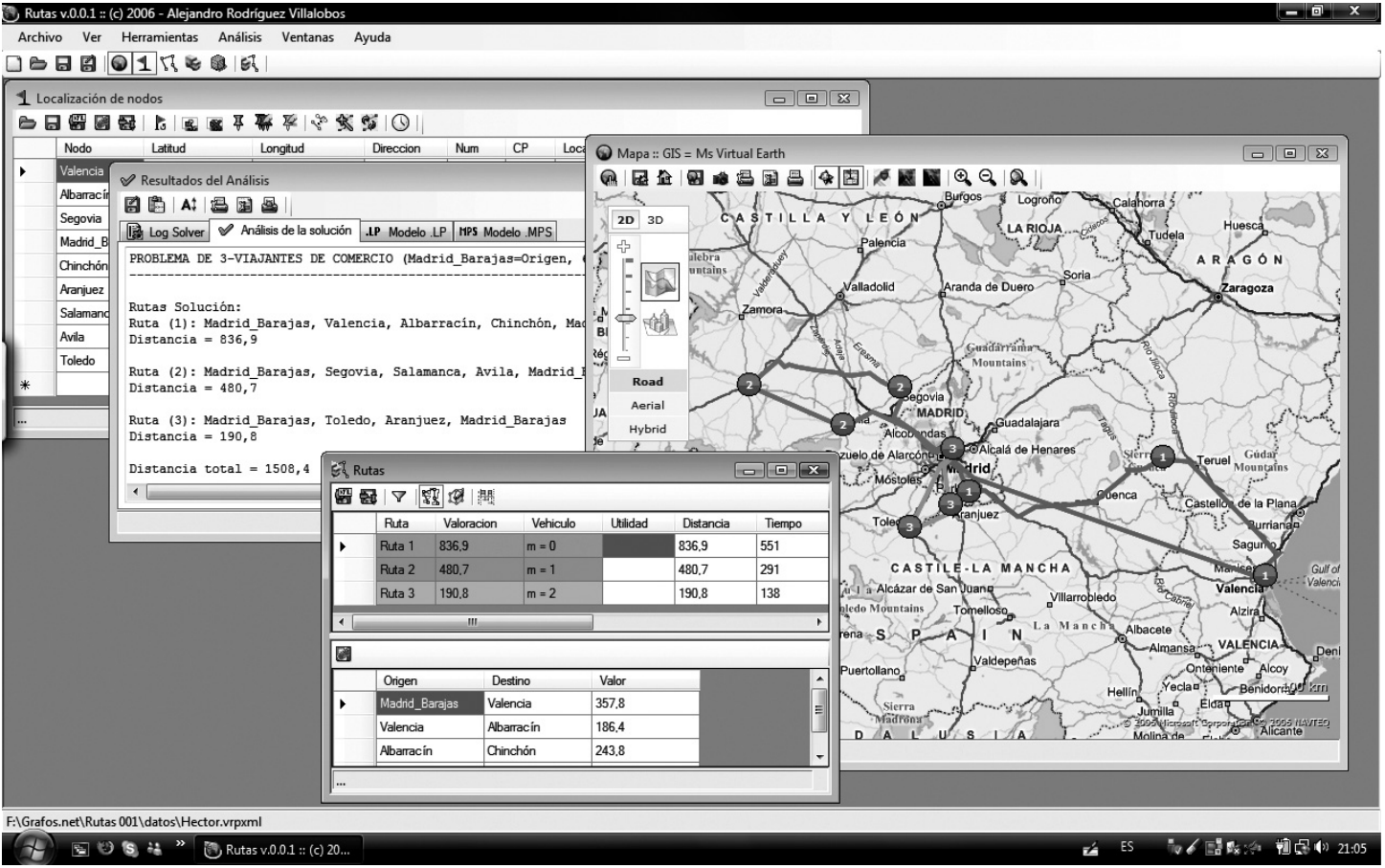


Tabla I

Análisis VRP implementados

\begin{tabular}{|c|c|}
\hline Análisis & Descripción \\
\hline DMP & Delivery Man Problem: Ciclo Hamiltoniano con inicio y fin en una localización seleccionada. \\
\hline SHP & Shortest Hamiltonian Path: Camino Hamiltoniano con inicio en la localización A y fin en la B. \\
\hline TSP & Traveling Salesman Problem: Problema del Viajante de Comercio. \\
\hline m-TSP & Problema de los m Viajantes de Comercio. \\
\hline CVRP & $\begin{array}{l}\text { CapacitedVehicle Routing Problem: Problema de Rutas con Vehículos Capacitados. Funciones objetivo: mín. } \\
\text { distancia, mín. núm. vehículos, mín. coste total (coste variable + coste fijo flota), etc. } \\
\text { Extensión del CVRP con limitaciones en el máximo número de clientes a visitar, y la máxima distancia (o } \\
\text { coste) requerido. CVRP single customer routes - con o sin la restricción de visitar I sólo cliente por vehículo }\end{array}$ \\
\hline DCVRP & $\begin{array}{l}\text { Distance-Constrained Capacited Vehicle Routing Problem: Problema de Rutas con Vehículos Capacitados } \\
\text { con limitaciones de distancia y/o clientes. }\end{array}$ \\
\hline BPP & $\begin{array}{l}\text { Asignación de vehículos a clientes para optimizar el uso de la flota y minimizar el coste de envío por unidad } \\
\text { de producto. }\end{array}$ \\
\hline VRPTW & $\begin{array}{l}\text { VRP with Time Window: Problema de Rutas de Vehículos Capacitados con Ventanas de Tiempo. Tiempos } \\
\text { de servicio (recogida y/o entrega). En la actualidad se están implementando extensiones a este análisis. }\end{array}$ \\
\hline
\end{tabular}

variable binaria $X_{i j k}$ indica si el vehículo $k$ tendrá una ruta utilizando el arco ij. Mientras, la variable binaria $Y_{i k}$ indica si el nodo i con demanda $d_{i}$ será atendido por el vehículo $k$ con capacidad $C_{k}$. Como se puede ver en la primera restricción cada nodo cliente deberá ser atendido únicamente por un vehículo (en el problema básico CVRP). En cambio del nodo origen 0 pueden partir todos los vehículos $K$ de la flota. $A$ continuación aparecen las restricciones de continuidad donde el vehículo que llegue a un cliente deberá también partir desde él. Tan sólo faltan las restricciones de capacidad: la demanda atendida por un vehículo (suma de $d_{i}$ ) no debe exceder su capacidad $C_{k}$. En el caso en que todos los vehículos tengan la misma capacidad, los valores $C_{k}$ serán iguales. Por último aparecen las condiciones de Miller y Tucker (1960), y la definición de variables binarias.

$$
\begin{array}{ll}
\min _{i \in V} \sum_{j \in V} c_{i j} \sum_{k=1}^{K} x_{i j k} & \\
\text { s.a. } & \\
\sum_{k=1}^{K} y_{i k}=1 & \forall i \in V \cdot \cdot\{0\} \\
\sum_{k=1}^{K} y_{0 k}=K & \\
\sum_{j \in V} x_{i j k}=\sum_{j \in V} x_{j i k}=y i k & \forall i \in V, k=1 . . K \\
\sum_{i \in V} d_{i} y_{i j} \leq c_{k} & \forall k=1 \ldots K
\end{array}
$$

$$
\begin{array}{ll}
\sum_{i \in S} \sum_{j \notin S} x_{i j k \geq} \geq y_{h k} & \forall S \quad V \cdot \cdot\{0\}, h \in S, k=1 \ldots K \\
x_{i j k} \in\{0,1\} & \forall i, j \in V, k=1 \ldots K \\
x_{i k} \in\{0,1\} & \forall i \in V, k=1 \ldots K
\end{array}
$$

En el tipo de problema más sencillo no se tiene en cuenta el horario de entrega o recogida en cada lugar de interés (ventanas horarias-VRPTW). La función objetivo podría ser: minimizar el número total de vehículos (o conductores) requeridos para dar servicio a todos los clientes, minimizar los costes fijos asociados con el uso de los vehículos (o los conductores), minimizar el coste total de transporte (coste fijo más variable de la ruta), balancear las rutas (por tiempo de viaje o carga de vehículo), minimizar las penalizaciones asociadas para un servicio parcial a los clientes, etc.

Para resolver los modelos de optimización, se cuenta con la ayuda de Ip_solve; se trata de un solver de programación linear entera mixta de licencia libre (LGPL-GNU lesser general public license). Este solucionador resuelve modelos de programación lineal (mixta) puros, con variables enteras/binarias, conjuntos semi-continuos y special ordered sets (SOS). No tiene límite en el tamaño de los modelos y acepta ficheros de entrada en formatos .lp y .mps. También se puede usar la librería del solver para ser llamada desde lenguajes de programación como: C,VB, .NET, Delphi, Excel, Java, etc. Está escrito en ANSI C 
y puede ser compilado para distintas plataformas como Linux y Windows. También se puede encontrar LUSOL, un sistema avanzado de factorización $L \cup$ y resolución de ecuaciones integrado en Ip_solve $v 5$ en el paquete bfp. También es importante citar la utilización de ficheros de datos auxiliares que son útiles para la resolución de problemas de rutas. Actualmente se utilizan dos formatos de fichero para la definición de modelos de programación lineal entera mixta (MILP), se trata de estándares (.lp, .mps) que sirven de pasarela entre la aplicación y el solucionador de problemas de optimización (Solver Ip_solve).

\subsubsection{Otras funciones}

Además de todo lo anterior, el software integra todo un conjunto de funciones que facilita la interacción con otros elementos de la cadena logística (proveedores y clientes, vehículos, otros sistemas: ERP-CRM, etc.) como por ejemplo:

- Localización de clientes, centros de tránsito y almacenes (geocoding, waypoints).

- Selección optimizada de vehículos (flota propia vs. subcontratada).

- Definición de zonas de distribución.

- Planificación de rutas de reparto y aprovisionamiento.

- Cálculo y gestión de distancias, tiempos y costes de transporte.

- Definición y análisis de ventanas horarias de entrega o recogida.
- Seguimiento de vehículos (GPS tracking).

- Intercambiar información sobre localizaciones e itinerarios con su navegador GPS.

- Generación de mapas, mejora de la documentación logística.

- Exportar información sobre localizaciones e itinerarios para otro software cartográfico (Google Earth, OziExplorer, GPS Visualizer, CompeGPS, Google Maps, GPS TrackMaker, etc.).

\section{Primeros resultados y conclusiones}

En este proyecto de acción-investigación se está teniendo la oportunidad de validar el desarrollo mediante frente a la resolución de problemas reales en empresa. Por cuestiones de confidencialidad no ha sido posible mostrar todavía en este artículo información detallada al respecto, pero cabe señalar la gran aceptación y bondad de los primeros resultados aportados por esta herramienta en las empresas piloto que han participado del proyecto (transporte internacional, distribución farmacéutica, vending, sector agro-alimentario). Como se puede observar en la siguiente (Figura 5), la herramienta está teniendo una gran acogida y muy buena valoración entre las empresas piloto. Un 69,23\% de las empresas consiguieron mejoras económicas en sus procesos de transporte del orden del 15-30\% respecto a su situación inicial.

En la actualidad el proyecto de investigación y desarrollo sigue en desarrollo. Se está abriendo el proceso de validación y financiación a nuevas empresas-piloto. Además se están programando nuevos modelos MILP para otros problemas VRP y algunas heurísticas

Figura 5

Resultados de la experiencia piloto del software Rutas.

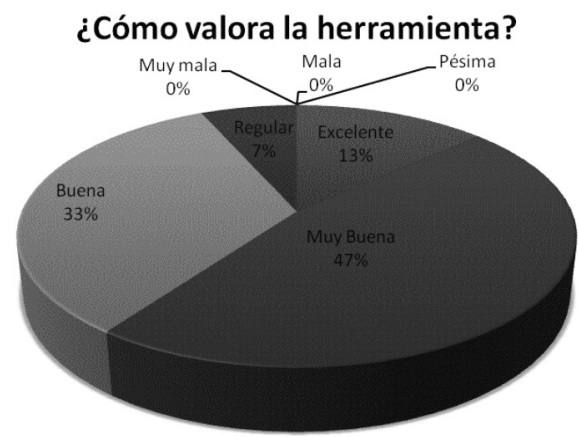

¿Qué mejora económica se ha logrado?

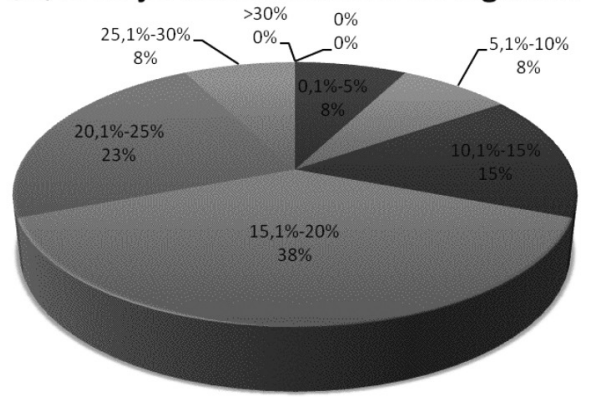


para casos concretos. Periódicamente se actualiza el solver de optimización con nuevas mejoras. Además se está mejorando y ampliando el módulo de generación de informes y análisis de las soluciones. En próximos artículos se espera poder presentar resultados más detallados y extensos sobre la valoración y utilización de esta herramienta en las empresas-piloto.

\section{Referencias}

BODIN, L., y GOLDEN, B. (1981). Classification in Vehicle-Routing and Scheduling, Networks, I I (2), pp. 97- I08.

DESROCHERS, M.; LENSTRA, J. K., y SAVELSBERGH, M.W. P. (1990). A Classification Scheme for Vehicle-Routing and Scheduling Problems, European
Journal of Operational Research, 46 (3), pp. 322-332.

MILLER, C.; TUCKER A., y ZEMLIN R. (1960). Integer programming formulations and traveling salesman problems, J. of the ACM, 7 326-329.

RODRIGUEZ, A. (2006). VRP-XML: lenguaje de marcas extensible para los problemas de rutas de vehículos. X Congreso de Ingeniería de Organización, Valencia.

TOTH, P., y VIGO, D. (200I). An overview of vehicle routing problems. In the Vehicle Routing Problem. Ed. Society for Industrial and Applied Mathematics. Philadelphia.

YEPES, V. (2002). Optimización heurística económica aplicada a las redes de transporte del tipo VRPTW. Escuela Técnica Superior de Ingenieros de Caminos, Canales y Puertos. Universidad Politécnica de Valencia. 